\title{
Prevalencia de quistes odontogénicos, Hospital Regional Valdivia entre los años 1990 y 2010
}

\section{Prevalence of odontogenic cysts, Hospital Regional Valdivia, between years 1990-2010}

\author{
Peters F I*, López G P*, Preisler E G**, Sotomayor C C***, Donoso Z M****, \\ Hernández V S*****
}

\section{RESUMEN}

Objetivo: Determinar la prevalencia de quistes odontogénicos en población atendida en el Hospital Regional Valdivia, en el periodo entre 1990-2010, según la última clasificación de lesiones quísticas de la Organización Mundial de la Salud (OMS), 2005. Esta investigación corresponde a un estudio descriptivo de corte transversal censal.

Metodología: Se revisaron informes de biopsias del Hospital Regional Valdivia (HRV), durante un periodo de 20 años (1990-2010), seleccionando los informes con diagnósticos histopatológicos de quistes odontogénicos. Las variables a analizar fueron: año de emisión del informe, género y edad del paciente, localización anatómica de la lesión, diagnóstico histopatológico. Los datos se almacenaron en planilla de base de datos para su análisis estadístico.

Resultados: De las 1.850 biopsias del territorio maxilofacial evaluadas, la prevalencia de Quistes Odontogénicos alcanzó un $11,9 \%$. Según tipo de quiste, los más prevalente fueron el quiste radicular (84,5\%), quiste dentígero $(14,1 \%$ y quiste residual $(0,9 \%)$. El de menor prevalencia fue el quiste de erupción (0,5\%). Un 52,7\% del total de quistes odontogénicos correspondieron al género femenino. El rango de edad de presentación va entre los 4 y los 81 años (promedio 31,7 $\pm 3,7$ años). El área anatómica más afectada es el maxilar superior con $65,5 \%$.

Conclusión: Los Quistes odontogénicos son entidades diagnosticadas histopatológicamente en forma variable durante el periodo de tiempo comprendido entre los años 1990 y 2010, en el HRV. Se presentan en mayor frecuencia en mujeres, sin predilección por género, afectando principalmente a la $3^{\text {a }}$ década de vida. La entidad quística mas prevalente fue el quiste radicular.

Palabras clave: Quiste odontogénico, quistes radiculares, quiste dentígero.

\section{SUMMARY}

Objective: Determine the prevalence of Odontological Cysts in the attended patients of Hospital Regional Valdivia, between 1990 and 2010, according to the World Health Organization (WHO) classification of tumour like lesions, 2005. This investigation corresponds to a descriptive cross-sectional study.

* $\quad$ Estudiante pregrado Odontología. Facultad de Medicina. Universidad Austral de Chile.

** Especialista en Cirugía y Traumatología Bucomaxilofacial. Hospital Regional Valdivia.

*** Médico Veterinario, Mg. Instituto Salud Pública. Universidad Austral de Chile.

**** Patólogo Oral, Subdepto. Dental, Hospital Regional Valdivia. Instituto Odontoestomatología. Facultad de Medicina. Universidad Austral de Chile.

***** Especialista en Endodoncia, MSc. Instituto de Odontoestomatología. Facultad de Medicina. Universidad Austral de Chile. 
Method: The biopsy reports of the Hospital Regional Valdivia, during a 20 years period (1990-2010), were analyzed, selecting those with Odontological Cysts histopathological diagnosis. The analyzed variables were: year of the biopsy report; patient's gender and age; anatomical location of the lesion and histopathological diagnosis. The extracted data were stored in a data base for descriptive statistical analysis.

Results: Within the 1850 biopsies found from the maxilofacial region, the Odontological Cysts prevalence was $11.9 \%$. According to the type of cyst, the most prevalent was Radicular/Periapical Cyst (84.5\%), followed by Dentigerous/Folicular Cyst (14.1\%), and then Residual Cyst (0.9\%). The least prevalent was Eruption Cyst (0.5\%). 52.7\% of all Odontological Cysts were found in women. The cysts's presentation among age ranges was between 4 and 81 years old (average $31.7 \pm 3.7$ years old). The most frequently affected anatomical region was the superior maxilla (65.5\%).

Conclusion: Odontological Cysts are histopathologically diagnosed lesions in varying ways between 1990 and 2010 at Hospital Regional Valdivia. Without gender predilection, they present most commonly affecting women, mainly those in the 3rd decade of life. The most common cystic lesion found was Radicular/Periapical Cyst..

Key words: Odontogenic Cysts, Radicular Cyst, Dentigerous Cyst.

Fecha de recepción: 14 de octubre de 2011.

Aceptado para publicación: 26 de noviembre de 2011.

Peters F I, López G P, Preisler E G, Sotomayor C C, Donoso Z M, Hernández V S. Prevalencia de quistes odontogénicos, Hospital Regional Valdivia entre los años 1990 y 2010. Av. Odontoestomatol 2012; 28 (6): 303-309.

\section{INTRODUCCIÓN}

Los quistes odontogénicos son cavidades patológicas recubiertas por epitelio, originados a partir de componentes epiteliales del aparato odontogénico o de restos celulares que quedan atrapados en el proceso de fusión dentro del hueso o en los tejidos gingivales periféricos (1).

De acuerdo a su etiología se pueden clasificar como "inflamatorios" o del "desarrollo". Ambos tipos de quistes son lesiones epiteliales, de crecimiento lento, expansivo y de comportamiento biológico benigno, sin embargo pueden alcanzar gran tamaño ante la ausencia de diagnóstico oportuno o tratamiento apropiado. Constituyen una de las principales causas de destrucción de los huesos maxilares (2). El diagnóstico de estas lesiones, basado fundamentalmente en los hallazgos histopatológicos, adquiere relevancia debido a las similares características clínicas y radiográficas que estos presentan. Es la histopatología la que finalmente determina el diagnóstico diferencial, justificando la elección de un plan de tratamiento y seguimiento adecuados (3).

La Organización Mundial de la Salud (OMS) definió, el año 2005, la última clasificación para quistes de origen odontogénico, basada en los conceptos actuales que definen al queratoquiste, en el cual se evidencia un comportamiento mas agresivo que otros quistes odontogénicos y por lo tanto incorporándolo en la categoría de tumores odontogénicos modificando así la clasificación hasta entonces utilizada desde 1992, y que fue propuesta por Kramer, Pindborg y Shear (1).

La prevalencia de los quistes odontogénicos varía según distribución geográfica, género y edad, entre otros. Estudios internacionales han reportado una prevalencia general dentro de las lesiones del territorio maxilofacial de un $10,4 \%$, siendo el quiste radicular el mas prevalente $(72,5 \%)(4,5)$. En cuanto a la edad de presentación, estudios mencionan 31 años promedio, con una prevalencia mayor en el género femenino (3). Cabe mencionar que tanto Tortorici et al. (2007) como De Souza et al. (2010), consideraron en sus estudios al queratoquiste, entidad que actualmente pertenece a la Clasificación de Lesiones Tumorales de la OMS, $2005(3,4)$.

En Chile, según estudio realizado por Ochsenius (2007), se ha reportado que estos quistes corresponderían a un $10 \%$ del total de muestras biopsia- 
das del territorio maxilofacial, incluyendo en su estudio al queratoquiste, con una proporción del $41,4 \%$ para hombres y un $58,6 \%$ para mujeres (2). Sin embargo, la literatura nacional es escasa e inexistente para la nueva región de los ríos.

El objetivo de esta investigación es determinar la prevalencia de quistes odontogénicos en población atendida en el Hospital Regional Valdivia (HRV) durante el periodo 1990-2010, según la última clasificación de lesiones tumorales de la OMS, año 2005.

\section{MATERIAL Y MÉTODO}

Se realizó un estudio descriptivo de corte transversal censal basado en la revisión de los archivos de registro de biopsias del subdepartamento de Anatomía Patológica del Hospital Base Valdivia realizadas durante el periodo comprendido entre enero de 1990 y diciembre del 2010. La población objetivo incluyó a todos aquellos pacientes que fueron diagnosticados mediante biopsia de alguna lesión en el territorio maxilofacial en dicho periodo.

Para la selección de los informes de biopsias se consideró la especificación de datos como género, año del diagnóstico y edad del paciente; además la presencia del diagnóstico histopatológico específico en cada uno de los casos y la zona anatómica de la cual fue obtenida la muestra biopsiada.

Se excluyeron todos aquellos informes de biopsias repetidos, incluso aquellas realizadas debido a recurrencias post tratamiento; informes ilegibles y aquellos que no tenían firma de un médico cirujano patólogo u odontólogo patólogo.

Se reevaluaron todos los diagnósticos histopatológicos de los informes según los conceptos actuales, señalados en la Clasificación de Quistes Odontogénicos de la OMS del año 2005. Para la determinación de la localización de los quistes odontogénicos, se consideraron dos áreas anatómicas: 1) Maxilar superior y 2) Mandíbula.

De un total de 202.421 informes de biopsias revisados, 1.850 corresponden a patologías del territorio bucomaxilofacial y 220 de ellas fueron considerados para este estudio.

Los datos extraídos fueron almacenados en planilla con los siguientes ítems: Número de ficha y de informe, año de emisión del informe, género y edad del paciente, localización de la lesión, diagnóstico clínico e histopatológico.

Los quistes odontogénicos fueron ordenados según prevalencia y distribución de acuerdo a las variables a evaluar. El análisis estadístico se realizó con el programa PASW STATISTICS 18, considerando la distribución de frecuencias absolutas y relativas de las variables estudiadas y determinación de medidas resumen con la media y de dispersión como la desviación estándar en aquellas variables que correspondiese. Se establecieron posibles relaciones entre las variables demográficas a medir y los tipos de quistes odontogénicos encontrados.

\section{RESULTADOS}

De las 202.421 biopsias revisadas, en el periodo 1990-2010, 220 corresponden a quistes maxilares, lo que equivale a un 1,9\%. Dentro de las biopsias del territorio maxilofacial, los quistes odontogénicos corresponden al 11,9\%. Según tipo de quiste, el más prevalente fue el radicular con un $84,5 \%$, seguido por dentígero con un $14,1 \%$ y luego por el quiste residual con un 0,9\%. Finalmente el de menor prevalencia es el de Erupción con un 0,5\% (Tabla 1).

\begin{tabular}{|c|c|c|c|c|c|c|}
\hline \multicolumn{7}{|c|}{$\begin{array}{c}\text { TABLA 1.- DISTRIBUCIÓN DE QUISTES } \\
\text { ODONTOGÉNICOS SEGÚN TIPO Y GÉNERO, } \\
\text { HRV 1990-2010 }\end{array}$} \\
\hline \multirow{2}{*}{$\begin{array}{l}\text { Diagnóstico } \\
\text { histopatológico }\end{array}$} & \multirow{2}{*}{$N$} & \multirow{2}{*}{$\%$} & \multicolumn{2}{|c|}{ Hombre } & \multicolumn{2}{|c|}{ Mujer } \\
\hline & & & $N$ & $\%$ & $\mathrm{~N}$ & $\%$ \\
\hline Quiste radicular & 186 & 84,5 & 86 & 46,3 & 100 & 53,7 \\
\hline Quiste dentígero & 31 & 14,1 & 17 & 54,9 & 14 & 45, \\
\hline $\begin{array}{l}\text { Quiste residual } \\
\text { Quiste de } \\
\text { erupción }\end{array}$ & 2 & 0,5 & 0 & 50 & 1 & 100 \\
\hline Total & 220 & 100 & 104 & 47,3 & 116 & 52,7 \\
\hline
\end{tabular}


De las lesiones biopsiadas, 104 (47,3\%) correspondieron al género masculino y 116 casos al género femenino (52,7\%). La relación hombre:mujer es de 0,9.

El rango de edad, según estratificación quinquenal, en que se presentaron estas lesiones abarca entre los 4 y los 81 años, con un promedio general de 31,7 $\pm 3,7$ años de edad. Un 58,2\% de los casos se concentran entre los 25 y 55 años de edad. La distribución según edad y género se presenta en la figura 1.

Según zona anatómica de presentación, un 34,5\% de los casos se encontraron en mandíbula y $65,5 \%$ en maxilar superior. La lesión quística más frecuentemente encontrada en ambos maxilares fue el quiste radicular, preferentemente en mujeres $(53,7 \%)$ con localización en maxilar superior en un $67,2 \%$ de los

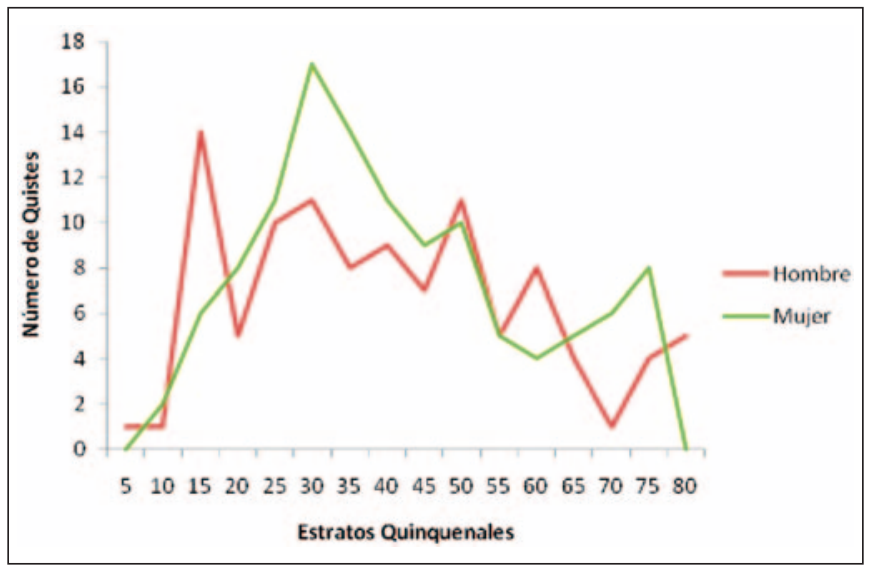

Fig. 1. Distribución de número de quistes según género y edad en estratos quinquenales. casos. El 62,3\% de los quistes radiculares se concentran entre los 25 y 55 años, con un peak de presentación entre los 25 y 29 años (13,4\%). La segunda lesión más prevalente fue el Quiste dentígero. Se presentó con mayor recurrencia en el maxilar superior $(54,8 \%)$ y en la población masculina $(54,9 \%)$. El $58 \%$ de estas lesiones se presentaron entre los $15 \mathrm{y}$ 35 años, con un peak de prevalencia para el rango de edad entre 20 y 24 años $(19,4 \%)$.

En cuanto al número de casos para el periodo en estudio hubo mayor diagnóstico de quistes odontogénicos durante el año 2010, con un total de 24 casos. Los años que presentaron menos biopsias fueron 1991 y 1995 con 2 casos cada uno (Figura 2).

\section{DISCUSIÓN}

En este estudio descriptivo, se logró revisar la totalidad de los informes de biopsias del periodo mencionado, con lo cual se creó una base de datos en relación al tema, la cual no existía para la comuna de Valdivia y Región de los Ríos, aportando información relevante a la estadística nacional en lo que a salud bucal se refiere. Sin embargo, se debe señalar como limitación propia de un estudio donde el diagnóstico fue realizado por varios profesionales en años previos, la dificultad de establecer una confiabilidad universal en los resultados entregados debido a la posible falta de calibración previa de los profesionales que diagnosticaron respectivamente las biopsias, además de la incorporación de otros profesionales a lo largo del periodo de tiempo que abarcó el estudio,

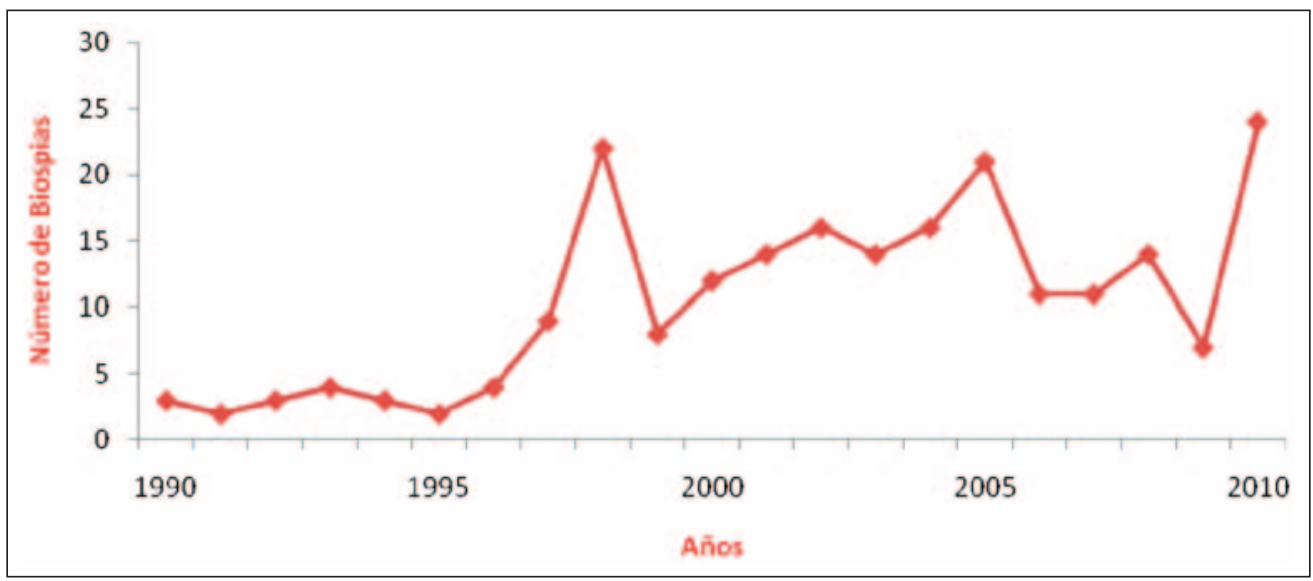

Fig. 2. Número de quistes odontogénicos diagnosticados por año, HRV 1990-2010. 
como fue el caso de un odontólogo patólogo al equipo, lo cual probablemente precisó aún más los hallazgos diagnósticos. Es recomendable, para una mayor validez externa de los datos obtenidos, que estas biopsias sean reevaluadas acorde a la clasificación actual por profesionales calibrados entre sí, ya que además fue necesario estandarizar los diagnósticos según criterios actuales vigentes. Debe considerarse el sesgo de información que presenta este tipo de estudio, donde los datos se obtuvieron de informes de biopsias que en algunas ocasiones no se encontraban completos careciendo de los datos mínimos requeridos para esta investigación, por lo cual no fueron consideradas en este estudio. Además está el sesgo de prevalencia, ya que no todas las lesiones clínicamente diagnosticadas como quistes odontogénicos se envían a biopsias, por lo cual la información encontrada corresponde sólo a los casos enviados a diagnóstico histopatológico.

La prevalencia encontrada para quistes odontogénicos fue de 11,9\% del total de lesiones biopsiadas de la cavidad oral, según la clasificación de Quistes Odontogénicos de la OMS año 2005, para el periodo entre 1990 y 2010 en el HRV. Similares resultados fueron registrados por Tortorici et al. (2007) en estudio realizado en Sicilia, Italia, donde se obtuvo una prevalencia general de $10,4 \%$ (4). En Brasil se reportaron prevalencias entre $6,6-9,9 \%$, mientras que Mosqueda et al. (2002), en México, describieron un $11,5 \%(1,6,7)$. En Santiago de Chile, el año 2007, Ochsenius et al., considerando en sus resultados al Queratoquiste, encontraron una prevalencia de 10\% del total de biopsias realizadas (2). En nuestro estudio se utilizó la última clasificación de Quiste Odontogénicos de la OMS, 2005, donde se excluye de los resultados al queratoquiste.

Según género, los quistes odontogénicos se presentan preferentemente en mujeres $(52,7 \%)$ con una relación hombre:mujer de 0,9. Este hallazgo es similar al 57,6\% encontrado por Avelar et al. (2009), el $53 \%$ descrito por Lima Gda et al. (2008) y finalmente por Tortorici et al. (2007), con un 54,4\% de mujeres afectadas; en ninguno de estos estudios se establecen diferencias significativas para esta variable $(4,6,7)$. En contraste, Varinauskas et al. (2006), reportaron una prevalencia mayor en hombres con un $53,5 \%$ al igual que Mosqueda et al. (2002), con un
$52 \%$ para el género masculino, tampoco estableciéndose diferencias estadísticamente significativas $(1,8)$.

En cuanto a la edad, el rango de presentación fue entre los 4 y 81 años, con un promedio de 31,7 $\pm 3,7$ años. El 58,2\% de las lesiones se presentaron entre los 25 y 55 años; dentro de estos el rango mas prevalente fue entre 25 y 29 años (12,7\%). Estos hallazgos son similares a los de Batista de Souza et al., quienes describieron un promedio de 31 años de edad (3). Núñez-Urrutia et al. (2010) encontraron un rango etario de distribución entre 7 y 83 años, con un promedio de 42 años y una mayor frecuencia entre la $3^{\mathrm{a}}$ y $4^{\mathrm{a}}$ década, al igual que Prockt et al. (2008) quienes reportaron una mayor prevalencia en la $4^{\circ}$ década $(5,9)$. Avelar et al. (2009) describieron un promedio de edad de 28,9 años y una prevalencia mayor en la $3^{a}$ década. (7). Según género, las edades entre 20 y 30 años en hombres y mujeres respectivamente son las más afectadas. Este hallazgo coincide con los resultados de Avelar et al. (2009) en Brasil en cuanto a la prevalencia en población femenina (7).

Según localización anatómica de las lesiones, un $65,5 \%$ de los quistes odontogénicos afectan el maxilar superior. Similares resultados son los obtenidos por Tortorici et al. (2008) y Batista de Souza et al. (2009), quienes señalan mayor prevalencia en similar área anatómica. $(3,4)$ Diferente situación es la encontrada por Núñez-Urrutia et al. (2010) quienes encontraron un 61,5\% para mandíbula (9). En Chile, Ochesnius et al. (2007) igualmente reportaron mayor prevalencia en la zona mandibular (2).

Respecto al diagnóstico histopatológico, la entidad más prevalente fue el quiste radicular con un $84,5 \%$. Similares hallazgos fueron reportados el 2006 por Meningaud et al. con una prevalencia de 53,5\% para esta lesión, al igual que Batista de Souza et al. (2009) con un $61,4 \%(3,10)$. Tortorici et al. (2008) describieron un $84,5 \%$ de prevalencia de este quiste en Italia, mientras que en Chile Ochsenius et al. (2007) registraron un $50,7 \%$ de estas lesiones $(2,4)$. Esta alta prevalencia de quistes radiculares puede ser explicada por el origen inflamatorio de los mismos, derivado de procesos infecciosos como caries dental, patología altamente prevalente en la población así como traumatismos dentales y tratamientos conservadores indicados por razones estéticas en las piezas dentarias afectadas (1). 
En cuanto a la presentación de quistes radiculares según género, es mayor en mujeres $(53,7 \%)$. Resultados similares fueron descritos por Tortorici et al. (2007), Prockt et al. (2008) y Mosqueda et al. (2002). $(1,4,5)$. En cuanto a la localización anatómica de las lesiones esta es mayor en maxilar superior $(67,2 \%)$. Mismos hallazgos señalan Batista de Souza et al. (2009), Prockt et al. (2008) y Tortorici et al. (2007), entre otros (3-5). El 62,3\% de los pacientes afectados se encuentran entre los 25 y 55 años, con un peak de prevalencia en la $3^{a}$ década. Tortorici et al. (2008), mencionan un promedio de edad de 35,6 años y Batista de Souza et al. (2010) 31,5 años promedio $(3,4)$. Otros estudios, como Prockt et al. (2008), describen el peak de prevalencia en la $4^{\mathrm{a}}$ década de vida (5). Según la literatura nacional, Ochsenius et al. (2007), describen en su estudio una mayor prevalencia para la $3^{a}$ década (2).

Meningaud et al. (2006), reportaron una prevalencia de 22,3\% para el quiste dentígero, ubicándolo en segundo lugar después del quiste radicular, coincidiendo con los hallazgos de este estudio donde este representa un $14,1 \%$ de los casos descritos (10). Similar tendencia se observa en estudios internacionales. Avelar et al. (2009) describieron un 30,7\% de lesiones atribuibles a quistes dentígeros, a diferencia de Varinauskas et al. (2006) quien menciona un 13,7\% $(7,8)$. Mosqueda et al. (2002) encontraron una mayor prevalencia de este en población masculina $(56,9 \%)$, al igual que los resultados obtenidos de este estudio (54,9\%) (1). En cuanto a localización anatómica del quiste dentígero, para el presente estudio estos fueron mayormente encontrados en el maxilar superior con 54,8\%. Similares resultados son descritos por Tortorici et al. (2007) (4). Los resultados obtenidos en cuanto a década de vida afectada coinciden con los expuestos en la literatura, describiéndose una mayor prevalencia en la segunda década, lo que es atribuible a la erupción de los terceros molares, piezas más frecuentemente afectadas por esta entidad patológica (2).

En tercer lugar de prevalencia se ubica el Quiste Residual $(0,9 \%)$ y finalmente el quiste de erupción $(0,05 \%)$. Misma tendencia es descrita en la literatura por Avelar et al. (2009) y Varinauskas et al. (2006) $(7,8)$.

En cuanto al número observado de casos para el periodo en estudio, hubo más biopsias de quistes odonto- génicos durante el año 2010. Estos datos no presentan gran relevancia puesto que el patrón de distribución de casos es irregular a lo largo del tiempo en estudio.

Los hallazgos encontrados en este estudio demuestran la importancia del diagnóstico de este tipo de lesiones, ya que los quistes odontogénicos son entidades de presentación frecuente en los maxilares, lo cual hace necesario el correcto examen por parte del clínico incluyendo la indicación de exámenes imagenológicos previo al tratamiento, ya sea este conservador o radical como la extracción de la pieza con el fin de obtener una aproximación concreta hacia el diagnóstico, enfatizando el oportuno y adecuado tratamiento de los mismos. Puesto que diversas entidades patológicas muestran similares imágenes radiográficas, es imprescindible la obtención de biopsias para la determinación del diagnóstico histopatológico.

En conclusión, los quistes odontogénicos son entidades diagnosticadas histopatológicamente en forma variable durante el periodo de tiempo comprendido entre los años 1990 y 2010, en el HRV. Estas lesiones se presentan con mayor frecuencia en mujeres, sin predilección por género, principalmente durante la $3^{a}$ década de vida. La entidad quística mas prevalente fue el quiste radicular, hallazgo respaldado por diversos estudios internacionales.

Se sugiere realizar otros estudios descriptivos en este ámbito, considerando la calibración de los profesionales que realicen los diagnósticos histopatológicos así como también indagar en aspectos relacionados con causalidad y significancia estadística entre las asociaciones probablemente establecidas en investigaciones futuras, que otorgarán mayor validez a esta línea de investigación.

\section{BIBLIOGRAFÍA}

1. Mosqueda-Taylor A, Irigoyen-Camacho ME, DiazFranco MA, Torres-Tejero MA. Odontogenic cysts. Analysis of 856 cases. Med Oral 2002 Mar-Apr; 7 (2):89-96.

2. Ochsenius G, Escobar E, Godoy L, Peñafiel C. Odontogenic cysts: analysis of 2,944 cases in Chile. Med Oral Patol Oral Cir Bucal 2007 Mar 1;12(2):E85-91. 
3. De Souza LB, Gordon-Nunez MA, Nonaka CF, de Medeiros MC, Torres TF, Emiliano GB. Odontogenic cysts: demographic profile in a Brazilian population over a 38-year period. Med Oral Patol Oral Cir Bucal 2010 Jul 1;15(4):e583-90.

4. Tortorici S, Amodio E, Massenti MF, Buzzanca ML, Burruano F, Vitale F. Prevalence and distribution of odontogenic cysts in Sicily: 19862005. J Oral Sci 2008 Mar;50(1):15-8.

5. Prockt AP, Schebela CR, Maito FD, Sant'AnaFilho M, Rados PV. Odontogenic cysts: analysis of 680 cases in Brazil. Head Neck Pathol 2008 Sep;2(3):150-6.

6. Lima Gda S, Fontes ST, de Araujo LM, Etges A, Tarquinio SB, Gomes AP. A survey of oral and maxillofacial biopsies in children: a single-center retrospective study of 20 years in Pelotas-Brazil. J Appl Oral Sci 2008 Nov-Dec;16(6): 397-402.

7. Avelar RL, Antunes AA, Carvalho RW, Bezerra PG, Oliveira Neto PJ, Andrade ES. Odontogenic cysts: a clinicopathological study of 507 cases. J Oral Sci 2009 Dec;51(4):581-6.

8. Varinauskas V, Gervickas A, Kavoliuniene O. Analysis of odontogenic cysts of the jaws. Medicina (Kaunas) 2006;42(3):201-7.

9. Nunez-Urrutia S, Figueiredo R, Gay-Escoda C. Retrospective clinicopathological study of 418 odontogenic cysts. Med Oral Patol Oral Cir Bucal 2010 Sep 1;15(5):e767-73.

10. Meningaud JP, Oprean N, Pitak-Arnnop P, Bertrand JC. Odontogenic cysts: a clinical study of 695 cases. J Oral Sci 2006 Jun;48(2):59-62.

\section{CORRESPONDENCIA}

Cristina Sotomayor C

Instituto Salud Pública,

Universidad Austral de Chile,

Valdivia, Chile

Correo electrónico: cristinasotomayor@uach.cl 